\title{
Removal of Ring Artifacts in Microtomography by Characterization of Scintillator Variations - an Extension to Phase Contrast
}

\author{
$\underline{\text { William Vågberg }}{ }^{1, *}$, Jakob C. Larsson ${ }^{1}$ and Hans M. Hertz ${ }^{1}$ \\ 1. Biomedical and X-ray Physics, KTH Royal Institute of Technology, Stockholm, Sweden. \\ * Corresponding author, william.vagberg@biox.kth.se
}

In all imaging applications, the avoidance or removal of artifacts is essential to achieve good image quality. One significant and classical error in micro-computed tomography $(\mu \mathrm{CT})$ is ring artifacts. They typically arise from variations in the detector response and appear as rings or half-rings around the axis of rotation. For a linear response, such errors are removed by a proper flat-field correction. However, in practice detectors contain imperfections and non-linearities, which cause errors to persist also in the flatfield corrected image. We have discovered remaining ring artifacts in $\mu \mathrm{CT}$ that arise from the combined effect of beam hardening and variations in the scintillator thickness [1]. In $\mu \mathrm{CT}$, the x-ray detecting component in the camera is commonly a screen of e.g. gadolinium oxysulphide powder. For thin screens, the relative thickness variations can become large, and therefore the response can vary largely with changes in spectrum. The combination of beam hardening and scintillator variations will induce non-linear changes to the image, which remain uncorrected by a conventional flat-field correction.

We have previously published an article on this phenomenon, together with a method to measure the scintillator thickness variations, and a method to correct for the variations in attenuation contrast projection images [1]. There, we used a model for the detector response $R$, considering the scintillator thickness:

$$
R\left(T_{o b j}(r), T_{S}(r), t\right)=g t \cdot \int S(E) e^{-\mu_{o b j}(E) T_{o b j}(r)} G\left(T_{S}(r), E\right) d E
$$

where $g$ is the detector gain, $t$ the exposure time, $r$ the transverse coordinate, $S(E)$ the spectrum, $\mu_{o b j}(E)$ the object attenuation coefficient, $T_{o b j}$ the object thickness, $G\left(T_{S}, E\right)$ the known scintillator yield function, depending on scintillator thickness and x-ray energy. Since $S(E)$ is measured and $G$ is known [2], the scintillator thickness can be calculated provided that the object is known. And vice versa, once it has been measured, the object thickness can be calculated, and thus the source of ring artifacts are removed. For further details please refer to Ref. 1.

In this conference contribution, we investigate how the correction method performs in propagationbased phase contrast [3]. The performance of the correction method completely relies on how well the incident spectrum to the detector can be estimated, using the measured detector intensity. The introduction of phase contrast undeniably makes this process more complicated, as the result in one pixel is no longer independent of other pixels. We propose an improved correction method for scintillator variations, considering not only the spectral variations arising from beam hardening, but also from phase contrast. We present this in theory and simulations. The method is kept simple, considering the limitation that it must be possible to use with large tomography datasets.

\section{Estimating the spectrum}

In Eq. 1, the spectrum incident to the detector is $S(E) e^{-\mu_{o b j}(E) T_{o b j}}$. This is correct in absorption contrast, provided that $\mu_{o b j}(E)$ is known. However, in propagation-based phase contrast, we must also consider the redistribution of intensity that occurs in the free-space propagation. We propose that ring 

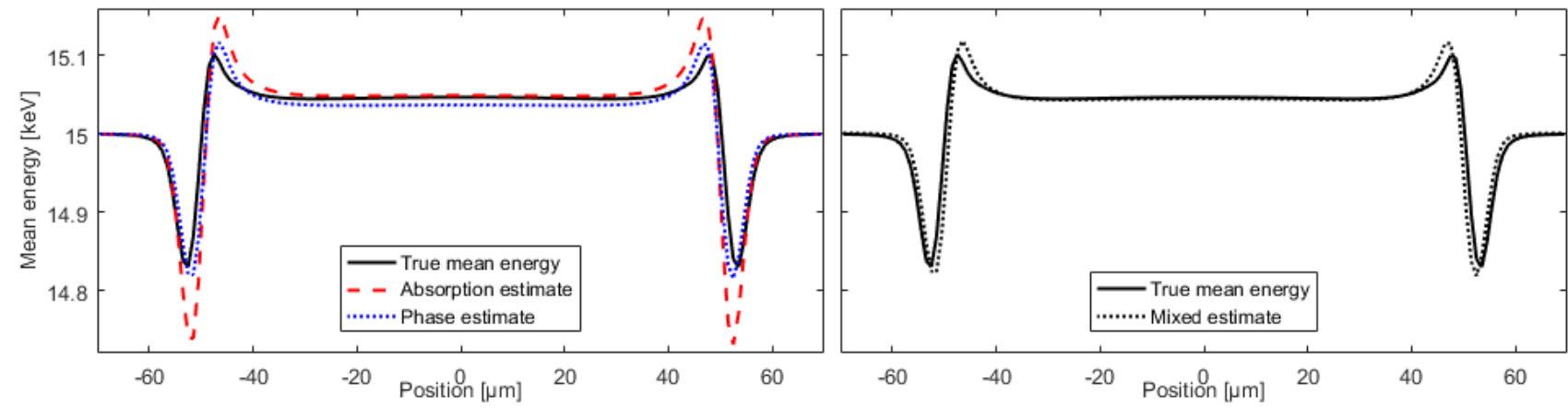

Figure. 1. Crosswise profiles of a simulated cylinder. True spectrum mean energy is taken directly from the simulation, whereas all estimates are based on the detected polychromatic image intensity.

artifact correction and phase retrieval is combined, in a method based on the Bronnikov-aided correction [4]. First, we modify Eq. 1 to also include the intensity redistribution due to the phase

$$
R\left(T_{o b j}(r), T_{S}(r), t\right)=g t \int S(E) e^{-\mu(E) T_{o b j}(r)}\left(1+z \delta(E) \nabla_{\perp}^{2} T_{o b j}(r)\right) G\left(T_{s}(r), E\right) d E .
$$

where $z$ the propagation distance and $\delta$ the refractive index decrement. Just as in the absorption-based correction [1], the solution is most easily found numerically, although here adding also the phase as another dimension. We propose the use of the Bronnikov-aided correction to separate the "absorption part" and "phase part". The spectrum is then calculated using only absorption or only phase, respectively. The parts are then recombined as in Eq. 2 to give a mixed estimate.

\section{Simulations}

The accuracy of the correction method depends on how well the spectrum is estimated. In this work, we limit ourselves to only study the estimation of the spectrum in the method. Simulations were performed with the software described in [5]. The simulations were done in a parallel beam geometry, with $0.1 \mathrm{~m}$ propagation distance to the detector. The detector was simulated with $1.0 \mu \mathrm{m}$ pixels, $5.0 \mu \mathrm{m}$ FWHM Gaussian point spread function, and ideal efficiency $\left(G\left(T_{s}, E\right)=1\right)$. To avoid aliasing, the simulation was oversampled to $0.1 \mu \mathrm{m}$. Polychromatic images were generated as sums of monochromatic images.

In this work, we simulated a $100 \mu \mathrm{m}$ diameter water cylinder as sample. 11 equal-weighted energies in the range 10-20 keV were simulated. In Fig. 1, we show a profile across the cylinder of the mean energy incident to the detector. The true mean energy is compared to estimates using the original absorptionbased method (Eq. 1), using a phase-only assumption (Eq. 2, $\mu=0$ ) and finally a mixed estimate using the method described above (Eq. 2). We have selected to show mean energies since they show the results from the method itself well, whereas intensity profiles would depend much more on the phase retrieval. From the presented results, we conclude that the mixed estimate performs similarly to the phase estimate close to the phase fringes, and similarly to the absorption estimate elsewhere. In summary, it improves the spectral estimate, which would thus improve the ring artifact correction method presented in [1].

[1] W Vågberg et al, Opt. Express 25 (2017), p. 23191-23198.

[2] R K Swank, Appl. Opt. 12 (1973), p. 1865-1870.

[3] S W Wilkins et al, Nature 384 (1996), p. 335-338.

[4] Y de Witte et al, J. Opt. Soc. Am. A 26 (2009), p. 890-894.

[5] U Lundström, PhD thesis, (KTH Royal Institute of Technology, Stockholm). 